\title{
The Relationship between Birth Timing, Circuit Wiring, and Physiological Response Properties of Cerebellar Granule Cells
}

S. Andrew Shustera,b,1, Mark J. Wagner ${ }^{\mathrm{a}, 1}$, Nathan Pan-Doh ${ }^{\mathrm{a}}$, Jing Ren ${ }^{\mathrm{a}, \mathrm{c}}$, Sophie M. Grutzner ${ }^{\mathrm{a}}$, Kevin T. Beier ${ }^{\mathrm{a}, \mathrm{d}}$, Tony Hyun Kim ${ }^{\mathrm{a}, \mathrm{e}}$, Mark J. Schnitzer ${ }^{\mathrm{a}, \mathrm{e}}$, Liqun Luo ${ }^{\mathrm{a},{ }^{*}}$

aDepartment of Biology and Howard Hughes Medical Institute, Stanford University, Stanford, 94305, California, USA

bNeurosciences Graduate Program, Stanford University, Stanford, 94305, California, USA

'MRC Laboratory of Molecular Biology, Cambridge University, Cambridge, CB2 0QH, UK

dDepartment of Physiology and Biophysics, University of California, Irvine, Irvine, 92697, California, USA

eDepartment of Applied Physics, Stanford University, Stanford, 94305, California, USA

${ }^{1}$ S.A.S. and M.J.W., equal contribution.

*To whom correspondence should be addressed. Email: 1luo@stanford.edu.

Keywords: cerebellum, granule cells, parallel fibers, birth timing, mossy fibers, rabies tracing, 2-photon $\mathrm{Ca}^{2+}$ imaging 


\begin{abstract}
Cerebellar granule cells (GrCs) are usually regarded as a uniform cell type that collectively expands the coding space of the cerebellum by integrating diverse combinations of mossy fiber inputs. Accordingly, stable molecularly or physiologically defined GrC subtypes within a single cerebellar region have not been reported. The only known cellular properties that distinguishes otherwise homogeneous GrCs is the correspondence between $\mathrm{GrC}$ birthtime and the depth of the molecular layer to which their axons (parallel fibers) project. To determine the role birth timing plays in $\mathrm{GrC}$ wiring and function, we developed genetic strategies to access early- and late-born GrCs. We initiated retrograde monosynaptic rabies virus tracing from control, early-born, and lateborn GrCs, revealing the different patterns of mossy fiber input to GrCs in vermis lobule 6 and simplex, as well as to early- and late-born GrCs of vermis lobule 6: sensory and motor nuclei provide more input to early-born GrCs, while basal pontine and cerebellar nuclei provide more input to late-born GrCs. In vivo multi-depth 2-photon $\mathrm{Ca}^{2+}$ imaging of parallel fibers of early- and late-born GrCs revealed representations of diverse task variables and stimuli by both populations, with differences in the proportions of parallel fibers encoding movement, reward anticipation, and reward consumption. Our results suggest neither organized parallel processing nor completely random organization of mossy fiber $\rightarrow$ GrC circuitry, but instead a moderate influence of birth timing on GrC wiring and encoding. Our imaging data also suggest that GrCs can represent general aversiveness, in addition to recently described reward representations.
\end{abstract}




\section{Significance Statement}

Cerebellar granule cells (GrCs) comprise the majority of all neurons in the mammalian brain and are usually regarded as a uniform cell type. However, the birth timing of an individual GrC dictates where its axon projects. Using viral-genetic techniques, we find that early- and late-born GrCs receive different proportions of inputs from the same set of input regions. Using in vivo multidepth 2-photon $\mathrm{Ca}^{2+}$ imaging of axons of early- and late-born $\mathrm{GrCs}$, we found that both populations represent diverse task variables and stimuli, with differences in the proportions of axons in encoding of a subset of movement and reward parameters. These results indicate that birth timing contributes to the input selection and physiological response properties of GrCs. 
lbody

\section{Introduction}

Cerebellar granule cells (GrCs) comprise the majority of neurons in the mammalian brain $(1,2)$. Each $\mathrm{GrC}$ receives only four excitatory inputs from mossy fibers, which originate in a variety of brainstem nuclei and the spinal cord, and the vast number of GrCs permits diverse combinations of mossy fiber inputs. Classical theories of cerebellar function have therefore proposed that GrCs function to integrate diverse, multimodal mossy fiber inputs and thus collectively expand coding space in the cerebellum (3-5). Until recently, studies have focused on the role of GrCs in implementing sparse coding of sensorimotor variables and stimuli (6-9). However, recent physiological studies of GrCs in awake, behaving animals highlight GrC encoding of cognitive signals in addition to sensorimotor signals (10-13). GrCs have also been recently shown to encode denser representations than expected by classical theory (10-12, 14-18), including a lack of dimensionality expansion under certain conditions (18).

Despite the vast number of GrCs, stable molecularly or physiologically defined GrC subtypes within a single cerebellar region or lobule have not been described (19-22). The only known axis along which spatially intermingled GrCs can be distinguished from each other is the depth of the molecular layer to which their parallel fiber axons (PFs) project, which is dictated by $\mathrm{GrC}$ lineage and birth timing $(23,24)$. Birth timing predicts the wiring and functional properties of diverse neuron types in many neural systems (25), including the neocortex $(26,27)$, other forebrain regions $(28,29)$, olfactory bulb $(30,31)$, and ventral spinal cord $(32,33)$. Furthermore, classic studies utilizing $\gamma$-irradiation at different times during rat postnatal development to ablate different cerebellar GrC and interneuron populations suggested that GrCs born at different times could contribute differentially to motor vs. action coordination (34). These observations also led to an as-of-yet untested hypothesis that mossy fibers arriving at different times during development could connect with different GrC populations. Could GrC birth timing be an organizing principle for information processing in the cerebellum?

Recent evidence and modeling point to the possibility of spatial clusters of co-activated PFs $(15,35)$, suggesting that GrCs born around the same time may disproportionally receive coactive mossy fiber inputs. However, another study using different methods and stimuli did not find differences in the physiological responses of early- and late-born GrCs to various sensorimotor stimuli (36). Here, we aimed to address the role of birth timing in $\mathrm{GrC}$ wiring and function. To do 
so, we developed strategies to gain genetic access to early- and late-born GrCs, as well as control GrCs not restricted by birth timing. We report the first monosynaptic input tracing to GrCs, finding differential mossy fiber inputs to GrCs in vermis lobule 6 and simplex, as well as different patterns of input to early- and late-born GrCs in vermis lobule 6. Finally, we performed in vivo multi-depth 2-photon $\mathrm{Ca}^{2+}$ imaging of PFs of early- and late- born GrCs during an operant task and presentation of a panel of sensory, appetitive, and aversive stimuli. We found differences in the proportions of early- and late-born GrCs encoding of a subset of movement and reward parameters. Together, these results reveal the contribution of $\mathrm{GrC}$ birth timing to their input wiring and diverse encoding properties. 


\section{Results}

Genetic Strategies for Accessing Birth Timing-defined Cerebellar Granule Cells. Cerebellar cortical circuit assembly occurs primarily during the first three postnatal weeks in the mouse, reaching full maturity shortly thereafter (37). At birth, GrC progenitors occupy the most superficial external granular layer (EGL), where they proliferate. Mossy fibers are morphologically recognizable after postnatal day 5 (P5), shortly after GrC progenitors have begun to exit mitosis (38). Newborn GrCs extend their axons as PFs into the developing molecular layer between the EGL and Purkinje cell bodies. Newborn GrC somata descend past the Purkinje cell layer (PCL) into the internal granular layer (IGL), giving rise to the granule cell layer in adults (39). Later-born GrCs stack their PFs superficially to those of earlier-born GrCs (24). As GrC neurogenesis proceeds, the EGL is gradually replaced by the molecular layer until P21, when GrC neurogenesis is complete (Fig. 1A).

To gain access to early- and late-born GrCs for anatomical profiling and physiological recordings, we developed genetic strategies for accessing birth timing-defined GrCs. We scoured the Allen Brain Atlas transgenic characterization database for CreER lines with expression specific to $\mathrm{GrC}$ progenitors and mature GrCs, but not mossy fiber origin sites in the brainstem (40). We identified two lines designed to mimic the expression pattern of the transcription factors Math1 (41), which is expressed selectively in GrC progenitors in the cerebellum (42), and Etv1 (43), which is expressed selectively in postmitotic cerebellar GrCs. To test our genetic strategies, we crossed Etv1-CreER and Math1-CreER mice to tdTomato Cre reporter mice (44). We injected 4hydroxytamoxifen (4OHT), the active metabolite of tamoxifen that drives CreER nuclear translocation, into Etv1-CreER;Ai14 mice at P7 and Math1-CreER;Ai14 mice at P13 to label earlyand late-born, respectively, GrCs. As predicted, these strategies resulted in PF fluorescence selectively in the deep and superficial portions of the molecular layer, respectively (Fig. 1B-E), allowing selective genetic access to early- and late-born GrCs. Furthermore, injecting 4OHT into Math1-CreER;Ai14 mice at P2 resulted in labeling of GrCs with PFs projecting to all depths of the molecular layer, indicating genetic access to $\mathrm{GrCs}$ not defined by birth timing as a control for comparison with birth timing-dependent results (Fig. 1F and G). Quantitative analysis of PF labeling showed that early- and late-born labeling strategies consistently allowed genetic access to specific and mutually exclusive populations of granule cells, but that the early-born strategy had a longer "tail" and thus more overlap with cells accessed via the control strategy (Fig. 1H). 
Differential Mossy Fiber Inputs to Granule Cells in Vermis Lobule 6 and Simplex. To our knowledge, mossy fiber inputs to GrCs from different precerebellar nuclei have not been quantitatively compared, largely due to longstanding technical limitations in performing input tracing from densely packed GrCs. Thus, prior to comparing inputs to early- and late-born GrCs, we first profiled the presynaptic inputs of mossy fibers to birth timing-unbiased GrC populations. We applied monosynaptic retrograde rabies virus (RV) tracing (Wickersham et al., 2007; Callaway and Luo, 2015) of mossy fiber input to GrCs of vermis lobule 6 and simplex. To do so, we crossed Math1-CreER mice to $R 26^{C A G-L S L-H T B}$ ( $R 26^{H T B}$ hereafter) mice (45), which express rabies G protein (B19SAD-RG) and the EnvA receptor TVA in a Cre-dependent manner. We injected Math1CreER;R2 $6^{H T B}$ mice with $4 \mathrm{OHT}$ at P2 and then injected G-deleted, GFP-expressing, EnvApseudotyped RV into vermis lobule 6 or simplex (bilateral) around P30 (P28-33; Fig. 2A). We sacrificed animals 5 days later and quantified GFP-labeled cells (Fig. 2B). This analysis revealed inputs from cells in many regions known to originate mossy fibers $(46,47)$, including the pons, various sensory and motor brainstem nuclei, and the cerebellar nuclei (Fig. 2C-M). In the absence of Cre, there were no retrogradely-labeled cells in the brainstem ( $\mathrm{n}=8$ mice); by contrast, the brainstems of experimental animals had thousands of input cells labeled (vermis: $2285 \pm 262$ cells; simplex: $2746 \pm 371$ cells), validating the specificity our transgenic-viral tracing methods (Fig. S2A).

We grouped inputs into four super-categories: pontine, motor, sensory, and cerebellar nuclei ( $\mathrm{CN}$; see Methods). Pontine input comprises input from the basal pons (BPN) and reticulotegmental nucleus of the pons (RtTg), both of which relay cortical information. Motor nuclei include the prepositus/hypoglossal nuclei (Pre/12N) and the gigantocellular (GRt), intermediate (IRt), parvicellular (PRt), lateral (LRt), and medullary (MRt) nuclei of the brainstem reticular system. Sensory nuclei include the interfascicular (IF5), primary sensory (Pr5), and spinal (Sp5) trigeminal nuclei, the medial $(\mathrm{MeVe})$ and lateral $(\mathrm{LaVe})$ vestibular nuclei, and the external cuneate nucleus $(\mathrm{ExC})$. Among inputs to both vermis lobule 6 and simplex, very few spinal cord and inferior olive (IO) cells ( $\ll 1 \%$, data not shown) were labeled, and so were not included in subsequent quantifications.

At the super-category level, quantitative analysis revealed that vermis lobule 6 received proportionally more pontine input than did simplex, while simplex received proportionally more 
input in aggregate from sensory brainstem nuclei than did lobule 6 (Fig. 2I). Quantitative analysis of individual nuclei revealed differential contributions to lobule 6 and simplex (Fig. 2J-M). For example, within the motor nuclei, lobule 6 received much more input from Pre/12N than did simplex, while simplex received more input from IRt and PRt than did lobule 6 (Fig. 2L). Among inputs from sensory nuclei, simplex received more input from trigeminal nuclei (IF5, Pr5, Sp5) than did lobule 6, while lobule 6 received more input from MeVe than did simplex (Fig. 2M). Finally, lobule 6 received a higher proportion of total inputs from pontine nuclei (Fig. 2K), in contradiction to classical conceptions of input to vermis coming mostly from the body rather than the cortex (48), but in line with recent multisynaptic tracing in rodents revealing substantial cerebral cortical input to lobule $6(18,49)$ and dorsal vermis generally $(50)$. These results also suggest that monosynaptic retrograde rabies virus tracing is sensitive enough to reveal quantitative differences in mossy fiber distributions.

Differential Mossy Fiber Inputs to Early- and Late-born Lobule 6 Granule Cells. We next performed monosynaptic retrograde RV tracing to reveal the distributions of mossy fiber input to early- and late-born GrCs. We crossed Math1-CreER and Etv1-CreER mice to R26 $6^{H T B}$ mice, injected Etv1-CreER;R26 $6^{H T B}$ mice and Math1-CreER;R26 $6^{H T B}$ with $4 \mathrm{OHT}$ at P7 and P13, respectively, to gain access to early- and late-born GrCs, followed by injecting vermis lobule 6 with RVdG-GFP-EnvA. We sacrificed mice 5 days later and analyzed rabies labeling results (Fig. $3 \mathrm{~A})$. We detected fewer input cells from early- and late-born samples than control samples (earlyborn: $476 \pm 54$ cells; late-born: $440 \pm 56$ cells), in accordance with the proportion of granule cells genetically accessed by each strategy (Fig. S3D).

Quantification revealed that late-born GrCs receive more pontine and CN input than earlyborn GrCs, and less input from motor and sensory nuclei (Fig. 3B). Early-born GrCs received similar absolute and within-super-category proportions of input as control GrCs from all input sources, possibly due to more overlap between early-born and control GrCs (Fig. 1H, 3B-E, and $\mathrm{S} 3)$. The increased pontine input received by late-born GrCs was due to an increase in BPN inputs (Fig. 3C). Late-born GrCs also received fewer Pre/12N, LRt, IF5, and ExC inputs than early-born GrCs (Fig. 3D and E). Overall, late-born GrCs received more of their within-pontine input from BPN than RtTg (Fig. S3A) and less of their within-motor nuclei input from GRt and LRt than early-born GrCs (Fig. S3B). These data suggest that late-born GrCs could represent a special 
population which receives a modified distribution of mossy fiber input or could reflect the greater specificity of our genetic access to late-born compared to early-born GrCs (Fig. 1H).

Taken together, these tracing results indicate that all precerebellar nuclei examined provide inputs to both early- and late-born GrCs, but with quantitative differences: sensory and motor nuclei provide more input to early-born GrCs, while basal pontine and cerebellar nuclei provide more to late-born GrCs.

Both Early- and Late-born Granule Cells Encode Diverse Signals. To address whether GrCs born at different times differ in their response properties towards diverse stimuli, we designed a preparation allowing near-simultaneous 2-photon imaging of $\mathrm{PF} \mathrm{Ca}^{2+}$ activity at two depths while mice perform an operant task (Fig. 4A-C), followed by presentation of a panel of appetitive, aversive, and neutral sensory stimuli (Fig. 4D; see Methods). To do so, we crossed Etv1-CreER mice to Ai148 mice, which express high levels of GCaMP6f via tTA2/TRE-mediated transcriptional activation in a Cre-dependent manner (51). We injected Etv1-CreER;Ail48 mice with 4OHT after P21 to drive GCaMP6f expression in a subset of PFs at all molecular layer depths without bias (Fig. 4A and Fig. S4A and B). We then trained water-restricted mice in an operant arm-reaching task with a randomly interspersed $20 \%$ of trials ending in reward (sucrose water) omission $(10,52)$. We imaged expert mice performing the operant task, followed by presentation of a stimulus panel containing visual and auditory stimuli, as well as a free reward and two aversive stimuli: orofacial air puff and tail shock; these five stimuli were repeatedly presented in a random order (Fig. 4D; see Methods). We imaged at two depths in the molecular layer, manually tuning the optical apparatus to identify the most superficial imaging field ( $\sim 10 \mu \mathrm{m}$ from the pial surface) and the deepest imaging field ( $\sim 10 \mu \mathrm{m}$ from the Purkinje cell layer) that we could access (Fig. $4 \mathrm{C})$. This provided near-simultaneous access to $\mathrm{Ca}^{2+}$ activity of PFs arising from late- and early-born GrCs (Movie 1). We then registered PFs and correlated their responses with different portions of the task and with each stimulus (Fig. 4E and F). Superficial and deep PFs had similar $\mathrm{Ca}^{2+}$ transient rates (Fig. S4C). In total, we imaged 5 mice over 19 sessions with independent fields-of-view and thus recorded from 831 superficial molecular layer PFs and 511 deep molecular layer PFs from vermis lobule 6 and simplex.

Overall, large fractions of both superficial and deep PFs exhibited responses to the operant task and free reward, smaller fractions responded to the aversive stimuli (orofacial air puff and tail 
shock), and even smaller fractions responded to the neutral auditory and visual stimuli (Fig. 4G). Both superficial and deep PFs encoded representations tiling the temporal scope of reward and reward omission trials in the operant task (Fig. 4H). Deep PFs, representing PFs of early-born GrCs, were positively modulated by movement and free reward in higher proportions than superficial PFs (Fig. 4I $\mathrm{I}_{1}$ and Fig. S4F), but no such differences were found in PFs negatively modulated by the task variables and stimuli (Fig. S4D). We also recovered PFs activated by reward, reward anticipation, and reward omission (10); the deep molecular layer had a higher proportion of reward anticipation PFs than the superficial molecular layer (Fig. 4 $\mathrm{I}_{2}$ ). Thus, while both superficial and deep PFs broadly represent task variables and stimuli, they do so in different proportions.

We also performed regression analysis using ten variables (pre-movement, post-movement, pre-operant reward, post-operant reward, reward omission, free reward, air puff, tail shock, tone, and visual stimuli) to uncover which variables best explain the dynamic activity levels of each PF (Fig. S5A and 5B). These analyses revealed that similar numbers of variables (regressors) contributed to activity changes in superficial and deep PFs (Fig. S5A), highlighting the potential for diverse, multimodal representations in PFs at each depth. This analysis also suggested that PFs tended to respond to clusters of different but potentially related task variables and stimuli, with two main clusters representing the task variables and stimuli (Fig. 4J). To estimate the dimensionality, or number of distinct modes of activity, in superficial and deep PF ensemble activity, we computed a principal components analysis on the complete imaging movies, and then tabulated the cumulative variance explained versus the number of principal components included in the reconstruction. Due to the difficulty of estimating dimensionality on a small number of cells, we alternatively computed the dimensionality on trial-averaged activity from all superficial or deep PFs in all imaging sessions. In both cases, a majority of the PF population activity variance was explained by $\sim 5-8$ principal dimensions (Fig. S5C).

Evidence that Some Granule Cells Encode General Aversion. Acquisition of the $\mathrm{Ca}^{2+}$ activity traces of PFs in response to diverse stimuli enabled us to investigate their encoding properties further. We found that PFs activated by orofacial air puff were often also activated by tail shock (Fig. 5A and B). Comprehensive correlation analyses revealed a strong correlation between PFs responding to air puff and PFs responding to tail shock (Fig. 5B $\left.1, B_{2}\right)$, representing the strongest 
correlation between the stimuli presented (Fig. 4J). By contrast, there was little correlation between free reward and air puff or tail shock (Fig. 5B $3-B_{6}$ ). These trends were evident in PFs at both depths. As orofacial air puff and tail shock are unlikely to activate the same primary sensory responses, these data suggest that some GrCs could encode generalized aversive stimuli or punishment.

\section{Discussion}

Birth timing is a determining factor for neuronal connectivity and function in a variety of systems from insects to mammals (25). In the mammalian neocortex, for example, birth timing dictates the cortical layers that glutamatergic excitatory neurons occupy $(26,27)$, which in turn predicts their connectivity patterns and functions (53). Birth timing also predicts the subtypes and cortical layers of GABAergic inhibitory neurons $(54,55)$ and the layer-specific axonal innervations of modulatory cholinergic neurons (29). In the cerebellar cortex, birth timing dictates the depth of the molecular layer to which the axons (PFs) of granule cells (GrCs) project (24). Even though PFs at different depths innervate the same Purkinje cells, whose dendrites span the entire molecular layer, there are at least two ways such spatially organized inputs could affect information processing. First, dendritic inputs at different distances from the soma can produce somatic membrane potential changes of different magnitudes and thus differentially affect action potential production $(56,57)$. Second, clustered synaptic inputs can summate nonlinearly to boost synaptic plasticity and transmission of synaptic potentials to the soma $(56,58-61)$. Thus, by mapping different mossy fiber populations to different depths of the Purkinje cell dendritic tree, GrC birth timing could modulate the impact of different inputs on cerebellar cortical output.

Our trans-synaptic tracing results indicate that early- and late-born GrCs receive inputs from the same collections of brainstem nuclei (Fig. 3). Our simultaneous in vivo imaging of deep and superficial PFs - corresponding to early- and late-born GrCs - indicate that they exhibit largely similar activity patterns during an operant task and in response to a battery of stimuli (Fig. 4). These data suggest that GrC input-output map is far from organized parallel processing, in which inputs from specific precerebellar nuclei are transmitted to specific depths of the molecular layer. Indeed, our imaging data largely agree with a recent report that did not detect differences in early- vs. late-born GrCs in their responses to sensorimotor stimuli (36). Together, these data are 
consistent with nonselective mossy fiber inputs onto GrCs born at different times, in line with the classic Marr-Albus framework (3, 4).

On the other hand, we identified quantitative differences between the distribution of mossy fiber inputs to early- vs. late-born GrCs, with brainstem sensory and motor nuclei contributing more inputs to early-born $\mathrm{GrCs}$ and pontine and cerebellar nuclei contributing more inputs to lateborn GrCs. Likewise, our imaging identified small but significant differences in the fraction of GrCs positively modulated by movement, reward anticipation, and reward consumption. These data rule out a completely stochastic mossy fiber $\rightarrow \mathrm{GrC}$ connectivity model. Future work is required to determine how differential mossy fiber inputs contribute to the differences in physiological response properties described here, and whether GrCs born at different times exhibit different activity patterns and functions in other behavioral paradigms.

The circuit architecture of the vertebrate cerebellar cortex has often been compared to that of the insect mushroom body $(5,62,63)$. Both circuits feature dimensionality expansion: a smaller number of mossy fibers provides input to a much larger number of GrCs in the cerebellar cortex; a small number olfactory projection neurons (PNs) provides input to a much larger number of Kenyon cells (KCs, the intrinsic neurons of the mushroom body) for representation of different combinations of olfactory cues. Anatomical and physiological studies have provided evidence for random $\mathrm{PN} \rightarrow \mathrm{KC}$ connections (64-66), even though birth timing is an important determining factor for both PNs $(67,68)$ and KCs (69). However, other physiological studies and more recent serial electron microscopic reconstructions have uncovered non-random connectivity (63, 70-72). Specifically, a subset of axons of PNs that represent food odors converge onto individual KCs more frequently than expected from a random connectivity model; this property can be used to enhance discrimination of commonly encountered and ethologically relevant stimuli (72). Furthermore, three major KC classes born at different times from a common neuroblast receive biased input from different PN types (63). Altogether, these studies suggest that the mushroom body circuit utilizes random connectivity within local regions themselves nested within structured organization at a larger scale $(63,72)$. Whether mossy fiber $\rightarrow \mathrm{GrC}$ connectivity follows similar rules awaits future investigation.

Our study also provides insights into GrC connectivity and coding unrelated to birth timing. Our description of mossy fiber inputs to vermis lobule 6 and simplex GrCs (Fig. 2) provide, to our knowledge, the first profiles of monosynaptic inputs to cerebellar GrCs. Notably, we found more 
pontine input to vermis lobule 6 than to simplex, a part of the cerebellar hemisphere (Fig. 2I). The vermis is classically considered a part of the spinocerebellar system, underscoring its reception of trigeminal and proximal somatosensory input; by contrast, the hemisphere is classically considered part of the cerebrocerebellar system, emphasizing its interconnections with the cerebral cortex (7375). Our results suggest a different view with regards to inputs to vermis vs. hemisphere, and are in line with multi-synaptic retrograde tracing studies from the vermis of monkeys (48), rats (50), and mice $(18,49)$. Together, these studies and ours argue against a simple, dichotomous conception of mossy fiber input to vermis vs. hemisphere.

Recent physiological recording studies in behaving animals have highlighted the role of cerebellum in reward processing (10-12, 76-80). Specifically, individual GrCs can encode reward delivery, omission, or expectation (10). We found that a substantial number of GrCs are activated by orofacial air puff and tail shock (Fig. 5), two aversive stimuli that use distinct sensory pathways. This finding suggests that GrCs may also encode generalized aversiveness, complementing their representation of reward and expanding our understanding of the cerebellum's role in cognitive processing (13). 


\section{Materials and Methods}

Mice. All procedures followed animal care and biosafety guidelines approved by Stanford University's Administrative Panel on Laboratory Animal Care and Administrative Panel of Biosafety in accordance with NIH guidelines. The day of birth was considered postnatal day 0 (P0). All mice were on CD1 and C57BL/6J mixed backgrounds. Transgenic lines Etv1-CreER (43), Math1-CreER (41), Ail4 (44), R26 HTB (45), and Ai148 (51) were used where indicated. Mice used to demonstrate genetic strategies were perfused/sacrificed between P28 and P32. Mice used in anatomical experiments were injected with rabies virus between P28 and P33 and perfused 5 days later. Mice used in imaging experiments were implanted with imaging windows at between 10 and 14 weeks of age. Both males and females were used in anatomical experiments; females were used in imaging experiments. Mice were housed in plastic cages with disposable bedding on a 12hour light-dark cycle with food and water available ad libitum until placed on water restriction.

4OHT production and injection. 4-Hydroxytamoxifen (4OHT) was prepared by dissolving in Chen oil (81). Math1-CreER;Ai14 and Math1-CreER;ROSA26 $6^{\text {HTB }}$ pups were injected intraperitoneally at P2 $(50 \mathrm{mg} / \mathrm{kg})$ or P13 (150 mg/kg). P13 Etvl-CreER;Ail4 or EtvlCreER;ROSA26 $6^{H T B}$ pups were injected intraperitoneally at P7 with $50-150 \mathrm{mg} / \mathrm{kg}$ 4OHT. EtvlCreER;Ai148 mice used in imaging experiments were injected intraperitoneally after P21 2-5 times with $150 \mathrm{mg} / \mathrm{kg} 4 \mathrm{OHT}$.

Immunostaining. Mice were deeply anesthetized using 2.5\% Avertin and perfused transcardially, first with $\sim 10 \mathrm{~mL}$ PBS then $\sim 25 \mathrm{~mL} 4 \%$ paraformaldehyde (PFA) in PBS. The fixed brains were dissected out and postfixed overnight at $4^{\circ} \mathrm{C}$ in $4 \%$ PFA in PBS. Brains were then immersed in sucrose 24-48 hours, embedded in Optimum Cutting Temperature (OCT, Tissue Tek), and sectioned on a cryostat (Leica).

For genetic strategy proof-of-principle experiments, $40-\mu \mathrm{m}$ sections of the cerebellar vermis were collected in PBS, washed twice in PBS for 10 minutes, incubated in 10\% normal donkey serum (NDS) in PBST (PBS with 0.3\% Triton X-100) for 2 hours at room temperature, and incubated in primary antibody (1:1,000 mouse anti-calbindin, Sigma; 1:500 rabbit anti-DsRed, Clontech; 1:1,000 guinea pig anti-vGluT1, Millipore) solution containing 5\% NDS in PBST for 2 overnights at $4^{\circ} \mathrm{C}$. Sections were then washed 3 times in PBST, each for 10 minutes; incubated in 
primary antibody solution containing 5\% NDS in PBST for 2 hours at room temperature; washed in PBST for 10 minutes, incubated in PBST with DAPI (1:10,000 of $5 \mathrm{mg} / \mathrm{mL}$, Sigma-Aldrich) for 30 minutes; then successively washed in PBST and PBS, each for 10 minutes. Sections were then mounted on Superfrost Plus slides, coverslipped with Fluoromount-G, and allowed to dry for at least 4 hours at room temperature before imaging.

For rabies tracing experiments, 60- $\mu \mathrm{m}$ sections encompassing the entire hindbrain (from AP -3.5 to -8.5) were collected onto Superfrost Plus slides in the order of sectioning. Slides were dried at room temperature overnight before further processing. Following overnight drying, slides were washed in PBST (PBS with 0.3\% Triton X-100) for 10 minutes, incubated in PBST with DAPI (1:10,000 of $5 \mathrm{mg} / \mathrm{mL}$, Sigma-Aldrich) for 30 minutes, then washed once in PBST and once in PBS, each for 10 minutes (all steps at room temperature). Slides were then dried and coverslipped with Fluoromount-G (Thermo Fisher Scientific). Slides were allowed to dry for at least 4 hours at room temperature before slide scanner imaging. Whole slides were then imaged with a 10x objective using a Leica Ariol slide scanner and a SL200 slide loader.

Fluorescence Imaging. Images were taken on a Zeiss LSM 780 laser-scanning confocal microscope (Carl Zeiss). To quantify the portion of the molecular layer innervated by genetically labeled GrCs, fluorescence intensity measurements were taken on unprocessed images in Fiji (ImageJ) and data were processed using custom MATLAB scripts. A 400-pixel-wide segmented line was drawn from the border of the deep molecular layer to the pial surface (the border of the superficial molecular layer), as defined by counter stains with calbindin and vGluT1, and average intensity values along the line were measured using the Plot Profile command. The intensity traces were interpolated into 100 bins from deep to superficial using custom code (MATLAB), and the traces from individual sections were normalized to a maximum of 100 . Numbers of sections were as follows: early-born strategy, 12 sections from 2 mice; late-born strategy, 12 sections from 2 mice; control strategy, 12 sections from 2 mice.

Rabies Virus Production and Injection. Pseudotyped (EnvA-coated) G-deleted rabies virus (RV) encoding expression of eGFP were produced following a published protocol (82). Mice were anesthetized with 1.5-2.0\% isoflurane and placed in a stereotaxic apparatus (Kopf Instruments). The following sets of coordinates (in mm) were used: $0.0 \mathrm{AP}, 0.0 \mathrm{ML},-0.3 \mathrm{DV}$ and $0.5 \mathrm{AP}, 0.0$ 
$\mathrm{ML},-0.3 \mathrm{DV}$ (relative to the posterior suture) for injections targeted to vermis lobule 6; and -2.0 $\mathrm{AP}, \pm 2.0 \mathrm{ML},-0.3 \mathrm{DV}$ (relative to lambda) for injections targeted to bilateral simplex. For injections targeting control GrCs in vermis lobule 6 and simplex (Fig. 2), 100 nL RVdG-eGFPEnvA was injected into each site. For injections targeting early- and late-born GrCs in vermis lobule 6 (Fig. 3), 300 nL RVdG-eGFP-EnvA was injected into each site. Following injection, mice were housed in a biosafety level 2 (BSL2) facility to allow for rabies transsynaptic spread and eGFP expression. All mice were injected between postnatal days 28 and 33 and sacrificed 5 days after RV injection.

Quantification of Rabies Tracing Results. Quantification of brainstem subregions relied on boundaries outlined in a mouse brain atlas (83). Anatomically contiguous regions with similar functions and lacking clear-cut anatomical boundaries were combined. For example, the prepositus (Pre) and hypoglossal nuclei $(12 \mathrm{~N})$ were combined due to their anatomical continuity, a lack of distinguishing anatomical markers in our histological preparation, and their co-involvement in motor coordination of facial muscles. The parvicellular reticular nucleus (PCRt) and its alpha part (PCRtA) were combined into PRt (parvicellular reticular nucleus); the intermediate reticular nucleus (IRt) and its alpha part (IRtA) were combined into IRt (intermediate reticular nucleus); the gigantocellular nucleus (Gi), including its alpha (GiA) and ventral (GiV) parts, and the lateral (LPGi) and dorsal (DPGi) paragigantocellular nuclei were combined into GRt (gigantocellular reticular nucleus); the dorsal (MdD) and ventral (MdV) parts of the medullary reticular nuclei were combined into MRt (medullary reticular nucleus). The magnocellular (MVeMC) and parvicellular (MVePC) parts of the vestibular nuclei and the vestibulocerebellar nucleus $(\mathrm{VeCb})$ were combined into $\mathrm{MeVe}$ (medial vestibular nucleus); while the lateral (LVe), spinal (SpVe), and superior ( $\mathrm{SuVe}$ ) vestibular subnuclei were combined into LaVe (lateral vestibular nucleus). The dorsomedial (Pr5DL) and ventrolateral (Pr5VL) parts of the principal sensory trigeminal nucleus were combined into $\operatorname{Pr} 5$ (principal sensory trigeminal nucleus); and the oral (Sp5O), dorsomedial (DMSp5), interpolar (Sp5I), and caudal (Sp5C) parts of the spinal trigeminal nucleus were combined into Sp5 (spinal trigeminal nucleus). Regions contributing under $0.75 \%$ total input, including the spinal cord, nucleus of the facial nerve $(7 \mathrm{~N})$, dorsal cochlear nucleus (DC), and inferior olive (IO) were not included in the final analysis due to the very low number of labeled cells in these regions. 
Tracing Statistics. Unpaired t tests with Bonferroni corrections and two-way ANOVAs with Tukey's multiple comparisons tests were performed using Prism 9 (GraphPad).

Window Implantation. Mice were anaesthetized using isoflurane (1.25-2.5\% in $0.7-1.3$ liter per minute of $\mathrm{O}_{2}$ ) during surgeries. Hair was removed from a small patch of skin, skin was cleaned, and an incision was made to remove the patch of skin. Connective tissue and muscle were then peeled back, and the skull was dried. A $3 \mathrm{~mm}$ diameter cranial window centered rostrocaudally over the post-lambda suture and $1.5 \mathrm{~mm}$ right of the midline was then exposed via drilling, positioning the window over cerebellar lobules VI and simplex. To seal the skull opening, a \#0 $3 \mathrm{~mm}$ diameter glass cover slip (Warner Instruments) was affixed to the bottom of a $3 \mathrm{~mm}$ outer diameter, $2.7 \mathrm{~mm}$ inner diameter stainless steel tube (McMaster) cut to $1 \mathrm{~mm}$ height. The glass/tube combination was stereotaxically inserted into the opening in the skull at an angle of $45^{\circ}$ from the vertical axis and $25^{\circ}$ from the AP axis. The window was then fixed in place and sealed with Metabond (Parkell). Next, a custom stainless steel head fixation plate was fixed to the skull with Metabond and dental cement (Coltene Whaledent). The $1.2 \mathrm{~mm}$ thickness fixation plate had a $5 \mathrm{~mm}$ opening to accommodate the stainless steel tube protruding from the window, and two lateral extensions to permit fixing the plate to stainless steel holding bars during imaging and behavior.

Operant Task. Briefly, mice were trained as described previously (10). During water restriction, mice were monitored daily for signs of distress, coat quality, eye closing, hunching and lethargy to ensure adequate water intake. Following two days of water restriction, mice were trained for 10-14 days for about 20-60 min daily, depending on performance and satiety. In both tasks, we recorded licking at $200 \mathrm{~Hz}$ using a capacitive sensor coupled to the metal water port which delivered approximately $\sim 6 \mu 14 \%$ sucrose water reward near the animal's mouth. During training and in all experiments, mice were head-fixed, with their bodies from the torso down in a custom printed optically transparent plastic tube. Mice learned to voluntarily initiate pushing the handle of a manipulandum. The robot constrained movement of the manipulandum to the forward axis. Handle position was controlled and monitored by two motors and encoders (Maxon B7A1F24007CF, containing DCX22S EB KL 24V motor with ENX 16 RIO 65536IMP encoder), 
and robotic control relied on nested feedback loops in FPGA $(10 \mathrm{kHz})$ and a real-time operating system computer $(1 \mathrm{kHz})$, both in a National Instruments cRIO chassis, as well as a Windows PC $(200 \mathrm{~Hz})$. The controllers were programmed in LabVIEW and permitted precise robotic positioning and application of forces to the handle with a $1 \mathrm{kHz}$ bandwidth to restrict motion as needed (52). The device recorded the handle position with a $200 \mathrm{~Hz}$ sampling rate and encoder resolution of $0.003 \mathrm{~mm}$ and permitted linear movements of maximum length $8 \mathrm{~mm}$, after which the trial terminated. Following a delay (1 s), a solenoid delivered the water reward. Following another delay $(1.7 \mathrm{~s})$ the handle began to return to the home position. This process took $2 \mathrm{~s}$ to complete, after which the mouse could initiate the next movement at any time. During reward omission trials, reward was withheld on a randomly interspersed $20 \%$ of trials, but never on two consecutive trials.

Imaging and Optics. All $\mathrm{Ca}^{2+}$ imaging was performed using a $40 \times 0.8 \mathrm{NA}$ objective (LUMPlanFLN-W, Olympus) and a custom two-photon microscope with an articulating objective arm. $920 \mathrm{~nm}$ laser excitation was delivered to the sample from a Ti:sapphire laser (MaiTai, Spectra Physics) at powers of around 40/50 $\mathrm{mW}$ at the superficial and deep imaging depths, respectively. ScanImage software (Vidrio Technologies) was used to control all image acquisition hardware. In order to image the superficial and deep molecular layer together, suitable depths for each were first identified. A z-piezo (P-725.4CD, Physik Instrumente) was used to lower the focal plane just below the pial surface until a full-field plane of the molecular layer was visualizable; this plane was recorded as the superficial depth. A full-field plane of the molecular layer just above the Purkinje cell layer (40 - $100 \mu \mathrm{m}$ below the superficial field-of-view) was then focused to and recorded as the deep depth. During imaging, ScanImage interleaved frame acquisitions at each depth by moving the z-piezo between frames, yielding a volume acquisition rate of $9.75 \mathrm{~Hz}$. At each depth, resonant galvanometers scanned a $240 \times 240 \mu \mathrm{m}$ field-of-view. To ensure alignment of the articulating objective to the glass window on the brain, a back-reflection procedure was performed. A low power visible red laser (CPS180, ThorLabs) co-aligned to the infrared beam onto the glass window was projected into the cerebellar window; the red back-reflection was then viewed via an iris placed on the objective port. The mouse and objective angles were positioned to center the back-reflection into the iris aperture. During image acquisition, slow axial drifts were compensated in real time by frequently comparing the acquired images to initial images and 
correcting using an objective $z$-piezo (P-725.4CD, Physik Instrumente). To align imaging data to behavioral data, the behavioral computer acquired the microscope's frame clock signal simultaneously with each mouse's behavioral data.

Stimulus Panel. A stimulus panel including delivery of free reward, orofacial air puff, tail shock, sliding tone and visual stimulus was designed. A total of 110 to 120 stimuli were presented over 15.4 minutes, with equal time intervals between presentation of successive stimuli. Free rewards consisted of a water reward of the same size as those of the operant task. Orofacial air puffs aimed at the mouse's left whisker pad and eye were triggered through an external custom-built stimulus delivery system; air puffs were tested before imaging and always elicited eyeblink. Tail shocks were administered via an electrical stimulator (model \#A320D, World Precision Instruments); electrode gel (Spectra 360, Parker Laboratories) was placed on the tail and the positive and negative leads (18-ga copper wire) were securely taped onto the middle portion of the tail $1 \mathrm{~cm}$ apart, and a $5 \mathrm{~V}$ pulse to the stimulator triggered delivery of an electrical current $(5 \mathrm{~mA}, 2 \mathrm{~s})$. The auditory tone stimulus consisted of a wav file encoding a 2-second-long tone rising from 1 $\mathrm{kHz}$ to $20 \mathrm{kHz}$ and then back down to $1 \mathrm{kHz}$ and was delivered via speakers from $1 \mathrm{~m}$ away from the mouse. The visual stimulus consisted of a $1 \mathrm{~s}$ duration moving vertical bar of blue light on a custom-built LED grid (Sparkfun WS2812B).

Image Processing. Files containing both superficial and deep images were first split by depth. Normcorre image registration software corrected rigid and piecewise-nonrigid lateral brain motion (84). Downsampled videos were manually inspected, and imaging sessions with significant brain motion/depth shift were discarded. Individual active PFs were identified in our imaging videos using automated cell sorting based on principal and independent component analyses (PCA/ICA). PFs corresponded to a weighted sum of pixels forming a spatial filter. Automated and manual segmentation and thresholding were used to truncate these filters down to individual PFs by eliminating spurious, disconnected components. Each PF's time-varying fluorescence trace was extracted by applying the spatial filter to the processed videos. Slow drift was removed from each trace by subtracting a tenth-percentile-filtered (15 s sliding window) version of the signal. Finally, each PF's fluorescence trace was $z$-scored to correct for differences in brightness between PFs; all fluorescence values were then reported in SD units. Data was aligned to the time of reward or 
stimulus delivery. For reward omission trials, data was aligned to the time at which reward would have been delivered following movement termination. The aligned fluorescence response of each PF was averaged across trials to produce the triggered averages shown.

Imaging Statistics. MATLAB (Mathworks) was used for all statistical tests. All comparisons of unpaired means of two groups used Wilcoxon rank sum tests. All comparisons of paired means of two groups used Wilcoxon signed rank tests. $p$ values were adjusted using the Holm-Bonferroni multiple comparisons correction.

Modulation Analysis. Time windows for modulation analysis were defined with reward (or, for reward omission trials, when reward would have been delivered) or stimulus delivery timepoints serving as timepoint $0.0 \mathrm{~s}$. To determine whether a PF was significantly modulated by a task variable/stimulus, we compared fluorescence before and after stimulus/variable. For each operant trial, we computed fluorescence for each cell averaged over the two time windows $[0.0,0.5] \mathrm{s}$ and $[-0.5,0.0] \mathrm{s}$, while for each stimulus delivery we used the time windows $[-1.0,0.0] \mathrm{s}$ and $[0,1.0]$ s. We then compared fluorescence in the two time windows. Significance for each cell was determined using a cutoff of $p=0.05$.

For the operant task, we defined reward-activated PFs as those whose fluorescence was significantly greater after than before reward, and significantly greater after reward than after reward omission ( $p<0.05$ for both comparisons). We defined reward anticipation PFs as those whose fluorescence was significantly greater after reward omission than after reward, and significantly greater just prior to reward than prior to movement ( $p<0.05$ for both comparisons). We defined reward omission PFs as those whose fluorescence was significantly greater after reward omissions than after reward $(p<0.05)$, excluding those previously defined as reward anticipation PFs. In analysis of these three PF response types, pre and post time windows were taken to be $[0.0,0.5] \mathrm{s}$ and $[-0.5,0.0] \mathrm{s}$.

Regression Analysis. To determine which task variables/stimuli predict PF activity, we used linear regression analysis to reproduce the time-varying fluorescence of each individual cell as a weighted sum of boxcar "indicator" functions corresponding to each behavioral variable of interest. The operant regressors corresponding to pre-movement, post-movement, pre-reward, and post- 
reward used boxcars of width $0.5 \mathrm{~s}$ just prior or subsequent to either movement or reward. The regressor corresponding to operant reward omission used a boxcar in the later period, [0.5, 1.5] s with respect to reward omission. The regressors corresponding to the stimuli-free reward, air puff, tail shock, tone, and visual—used boxcars at $[0,1] \mathrm{s}$ with respect to stimulus onset. These coefficients were defined as significant if $p<0.01$.

Principal Components Analysis. To quantify the number of dimensions in the PF imaging data, we computed principal components analysis (PCA). For a single-session and single-trial analysis, we took the entire $\mathrm{T} \times \mathrm{N}$ movie matrix ( $\mathrm{T}$ timepoints, $\mathrm{N}$ parallel fibers) and computed PCA. For an across-sessions, trial-averaged analysis, for each of the 6 contexts (operant reward, free reward, puff, shock, tone, visual) we computed the trial-averaged response (consisting of $\mathrm{T}_{\text {trial }}$ timepoints) for all PFs, then concatenated all trial types into a single $6 \mathrm{~T}_{\text {trial }} \times \mathrm{N}_{\text {total }}$ matrix (where $\mathrm{N}_{\text {total }}$ is the total number of PFs recorded from all sessions and mice). For either single-trial or trial-averaged, we computed PCA on the data matrix and tabulated the cumulative variance explained as a rising function of the number of principal components included.

Event detection. $\mathrm{Ca}^{2+}$ transients were defined as fluorescence local maxima (highest peak within $0.5 \mathrm{~s}$ ) of minimum magnitude $1.5 \mathrm{z}$-scores.

Data availability. Reagents and code are available upon reasonable request to the corresponding author.

\section{Acknowledgements}

We thank the Luo lab, especially H. Li, J. Lui, and Y. Takeo, for feedback on the project and comments on the manuscript; M. Molacavage for administrative assistance; C. Manalac for assistance with genotyping; and A. Joyner for the Math1-CreER mice and M. Goulding for R26 $6^{H T B}$ mice. S.A.S. was supported by a National Science Foundation Graduate Research Fellowship and a Regina Casper Stanford Graduate Fellowship. M.J.W. was supported by a Career Award at the Scientific Interface from the Burroughs Wellcome Fund. N.P-D. was supported by the Biology Summer Undergraduate Research Program and an Undergraduate Advising and Research Major Grant. K.T.B. was supported by the Brain and Behavior Research Foundation (NARSAD 26845). 
L.L. is an investigator of the Howard Hughes Medical Institute. This work was supported by National Institutes of Health grants K99-DA041445 (to K.T.B.) and R01-NS080835 (to L.L.).

\section{Author Contributions}

S.A.S. conceived the project, designed and performed all experiments and analyzed data. M.J.W. contributed substantially to all aspects of the 2-photon imaging experiments, including design, implementation, and data analysis. N.P-D. assisted with pilot tracing experiments. J.R., K.T.B. and S.M.G. provided viral reagents. T.H.K. and M.J.S. provided critical equipment and assistance with the 2-photon imaging experiments. S.A.S., M.J.W., and L.L. wrote the paper. L.L. supervised the project.

\section{Competing Interests}

The authors declare no competing financial interests. 


\section{References}

1. Lange W (1975) Cell number and cell density in the cerebellar cortex of man and some other mammals. Cell Tissue Res 157(1):115-124.

2. Herculano-Houzel S (2010) Coordinated scaling of cortical and cerebellar numbers of neurons. Front Neuroanat 4:12.

3. Marr D (1969) A theory of cerebellar cortex. J Physiol (Lond) 202(2):437-470.

4. Albus JS (1971) A theory of cerebellar function. Math Biosci 10(1-2):25-61.

5. Litwin-Kumar A, Harris KD, Axel R, Sompolinsky H, Abbott LF (2017) Optimal degrees of synaptic connectivity. Neuron 93(5):1153-1164.e7.

6. Sawtell NB (2010) Multimodal integration in granule cells as a basis for associative plasticity and sensory prediction in a cerebellum-like circuit. Neuron 66(4):573-584.

7. Huang C-C, et al. (2013) Convergence of pontine and proprioceptive streams onto multimodal cerebellar granule cells. Elife 2:e00400.

8. Ishikawa T, Shimuta M, Häusser M (2015) Multimodal sensory integration in single cerebellar granule cells in vivo. Elife 4.

9. Chabrol FP, Arenz A, Wiechert MT, Margrie TW, DiGregorio DA (2015) Synaptic diversity enables temporal coding of coincident multisensory inputs in single neurons. Nat Neurosci 18(5):718-727.

10. Wagner MJ, Kim TH, Savall J, Schnitzer MJ, Luo L (2017) Cerebellar granule cells encode the expectation of reward. Nature 544(7648):96-100.

11. Giovannucci A, et al. (2017) Cerebellar granule cells acquire a widespread predictive feedback signal during motor learning. Nat Neurosci 20(5):727-734.

12. Becker MI, Person AL (2017) Cerebellar granule cells expand their talents. Nat Neurosci 20(5):633-634.

13. Wagner MJ, Luo L (2020) Neocortex-Cerebellum Circuits for Cognitive Processing. Trends Neurosci 43(1):42-54.

14. Badura A, De Zeeuw CI (2017) Cerebellar granule cells: dense, rich and evolving representations. Curr Biol 27(11):R415-R418.

15. Gilmer JI, Person AL (2017) Morphological constraints on cerebellar granule cell combinatorial diversity. J Neurosci 37(50):12153-12166.

16. Knogler LD, Markov DA, Dragomir EI, Śtih V, Portugues R (2017) Sensorimotor Representations in Cerebellar Granule Cells in Larval Zebrafish Are Dense, Spatially Organized, and Non-temporally Patterned. Curr Biol 27(9):1288-1302.

17. Gilmer JI, Person AL (2018) Theoretically sparse, empirically dense: new views on cerebellar granule cells. Trends Neurosci 41(12):874-877.

18. Wagner MJ, et al. (2019) Shared Cortex-Cerebellum Dynamics in the Execution and Learning of a Motor Task. Cell 177(3):669-682.e24.

19. Carter RA, et al. (2018) A Single-Cell Transcriptional Atlas of the Developing Murine Cerebellum. Curr Biol 28(18):2910-2920.e2.

20. Gupta I, et al. (2018) Single-cell isoform RNA sequencing characterizes isoforms in thousands of cerebellar cells. Nat Biotechnol.

21. Rosenberg AB, et al. (2018) Single-cell profiling of the developing mouse brain and spinal cord with split-pool barcoding. Science 360(6385):176-182.

22. Kozareva V, et al. (2020) A transcriptomic atlas of the mouse cerebellum reveals regional specializations and novel cell types. BioRxiv. 
23. Zong H, Espinosa JS, Su HH, Muzumdar MD, Luo L (2005) Mosaic analysis with double markers in mice. Cell 121(3):479-492.

24. Espinosa JS, Luo L (2008) Timing neurogenesis and differentiation: insights from quantitative clonal analyses of cerebellar granule cells. J Neurosci 28(10):2301-2312.

25. Li H, Shuster SA, Li J, Luo L (2018) Linking neuronal lineage and wiring specificity. Neural Dev 13(1):5.

26. Angevine JB, Sidman RL (1961) Autoradiographic study of cell migration during histogenesis of cerebral cortex in the mouse. Nature 192:766-768.

27. Molyneaux BJ, Arlotta P, Menezes JRL, Macklis JD (2007) Neuronal subtype specification in the cerebral cortex. Nat Rev Neurosci 8(6):427-437.

28. Deguchi Y, Donato F, Galimberti I, Cabuy E, Caroni P (2011) Temporally matched subpopulations of selectively interconnected principal neurons in the hippocampus. Nat Neurosci 14(4):495-504.

29. Allaway KC, et al. (2020) Cellular birthdate predicts laminar and regional cholinergic projection topography in the forebrain. Elife 9.

30. Imamura F, Ayoub AE, Rakic P, Greer CA (2011) Timing of neurogenesis is a determinant of olfactory circuitry. Nat Neurosci 14(3):331-337.

31. Imamura F, Greer CA (2015) Segregated labeling of olfactory bulb projection neurons based on their birthdates. Eur J Neurosci 41(2):147-156.

32. Tripodi M, Stepien AE, Arber S (2011) Motor antagonism exposed by spatial segregation and timing of neurogenesis. Nature 479(7371):61-66.

33. Tripodi M, Arber S (2012) Regulation of motor circuit assembly by spatial and temporal mechanisms. Curr Opin Neurobiol 22(4):615-623.

34. Pellegrino LJ, Altman J (1979) Effects of differential interference with postnatal cerebellar neurogenesis on motor performance, activity level, and maze learning of rats: a developmental study. J Comp Physiol Psychol 93(1):1-33.

35. Wilms CD, Häusser M (2015) Reading out a spatiotemporal population code by imaging neighbouring parallel fibre axons in vivo. Nat Commun 6:6464.

36. Markwalter KH, Yang Y, Holy TE, Bonni A (2019) Sensorimotor coding of vermal granule neurons in the developing mammalian cerebellum. J Neurosci 39(34):6626-6643.

37. Leto K, et al. (2016) Consensus paper: cerebellar development. Cerebellum 15(6):789828.

38. Mason CA, Gregory E (1984) Postnatal maturation of cerebellar mossy and climbing fibers: transient expression of dual features on single axons. J Neurosci 4(7):1715-1735.

39. Altman J (1972) Postnatal development of the cerebellar cortex in the rat. I. The external germinal layer and the transitional molecular layer. J Comp Neurol 145(3):353-397.

40. Lein ES, et al. (2007) Genome-wide atlas of gene expression in the adult mouse brain. Nature 445(7124):168-176.

41. Machold R, Fishell G (2005) Math1 is expressed in temporally discrete pools of cerebellar rhombic-lip neural progenitors. Neuron 48(1):17-24.

42. Ben-Arie N, et al. (1997) Math1 is essential for genesis of cerebellar granule neurons. Nature 390(6656):169-172.

43. Taniguchi H, et al. (2011) A resource of Cre driver lines for genetic targeting of GABAergic neurons in cerebral cortex. Neuron 71(6):995-1013.

44. Madisen L, et al. (2010) A robust and high-throughput Cre reporting and characterization system for the whole mouse brain. Nat Neurosci 13(1):133-140. 
45. Li Y, et al. (2013) Molecular layer perforant path-associated cells contribute to feedforward inhibition in the adult dentate gyrus. Proc Natl Acad Sci USA 110(22):9106-9111.

46. Llinás RR, Walton KD, Lang EJ (2004) in The synaptic organization of the brain, ed Shepherd GM (Oxford University Press), pp 271-310.

47. Fu Y, Tvrdik P, Makki N, Paxinos G, Watson C (2011) Precerebellar cell groups in the hindbrain of the mouse defined by retrograde tracing and correlated with cumulative Wnt1cre genetic labeling. Cerebellum 10(3):570-584.

48. Coffman KA, Dum RP, Strick PL (2011) Cerebellar vermis is a target of projections from the motor areas in the cerebral cortex. Proc Natl Acad Sci USA 108(38):16068-16073.

49. Henschke JU, Pakan JM (2020) Disynaptic cerebrocerebellar pathways originating from multiple functionally distinct cortical areas. Elife 9.

50. Suzuki L, Coulon P, Sabel-Goedknegt EH, Ruigrok TJH (2012) Organization of cerebral projections to identified cerebellar zones in the posterior cerebellum of the rat. $J$ Neurosci 32(32):10854-10869.

51. Daigle TL, et al. (2018) A Suite of Transgenic Driver and Reporter Mouse Lines with Enhanced Brain-Cell-Type Targeting and Functionality. Cell 174(2):465-480.e22.

52. Wagner MJ, Savall J, Kim TH, Schnitzer MJ, Luo L (2020) Skilled reaching tasks for head-fixed mice using a robotic manipulandum. Nat Protoc 15(3):1237-1254.

53. Douglas RJ, Martin KAC (2004) Neuronal circuits of the neocortex. Annu Rev Neurosci 27:419-451.

54. Miyoshi G, Butt SJB, Takebayashi H, Fishell G (2007) Physiologically distinct temporal cohorts of cortical interneurons arise from telencephalic Olig2-expressing precursors. $J$ Neurosci 27(29):7786-7798.

55. Taniguchi H, Lu J, Huang ZJ (2013) The spatial and temporal origin of chandelier cells in mouse neocortex. Science 339(6115):70-74.

56. Stuart G, Spruston N, Häusser M (2016) Dendrites (books.google.com).

57. De Schutter E, Bower JM (1994) Simulated responses of cerebellar Purkinje cells are independent of the dendritic location of granule cell synaptic inputs. Proc Natl Acad Sci USA 91(11):4736-4740.

58. Wang SS, Denk W, Häusser M (2000) Coincidence detection in single dendritic spines mediated by calcium release. Nat Neurosci 3(12):1266-1273.

59. Marcaggi P, Attwell D (2005) Endocannabinoid signaling depends on the spatial pattern of synapse activation. Nat Neurosci 8(6):776-781.

60. Rancz EA, Häusser M (2006) Dendritic calcium spikes are tunable triggers of cannabinoid release and short-term synaptic plasticity in cerebellar Purkinje neurons. J Neurosci 26(20):5428-5437.

61. Takahashi N, et al. (2012) Locally synchronized synaptic inputs. Science 335(6066):353356.

62. Stevens CF (2015) What the fly's nose tells the fly's brain. Proc Natl Acad Sci USA 112(30):9460-9465.

63. Li F, et al. (2020) The connectome of the adult Drosophila mushroom body provides insights into function. Elife 9.

64. Masuda-Nakagawa LM, Tanaka NK, O’Kane CJ (2005) Stereotypic and random patterns of connectivity in the larval mushroom body calyx of Drosophila. Proc Natl Acad Sci USA 102(52):19027-19032.

65. Murthy M, Fiete I, Laurent G (2008) Testing odor response stereotypy in the Drosophila 
mushroom body. Neuron 59(6):1009-1023.

66. Caron SJC, Ruta V, Abbott LF, Axel R (2013) Random convergence of olfactory inputs in the Drosophila mushroom body. Nature 497(7447):113-117.

67. Jefferis GS, Marin EC, Stocker RF, Luo L (2001) Target neuron prespecification in the olfactory map of Drosophila. Nature 414(6860):204-208.

68. Yu H-H, et al. (2010) A complete developmental sequence of a Drosophila neuronal lineage as revealed by twin-spot MARCM. PLoS Biol 8(8).

69. Lee T, Lee A, Luo L (1999) Development of the Drosophila mushroom bodies: sequential generation of three distinct types of neurons from a neuroblast. Development 126(18):4065-4076.

70. Gruntman E, Turner GC (2013) Integration of the olfactory code across dendritic claws of single mushroom body neurons. Nat Neurosci 16(12):1821-1829.

71. Zheng Z, et al. (2018) A Complete Electron Microscopy Volume of the Brain of Adult Drosophila melanogaster. Cell 174(3):730-743.e22.

72. Zheng Z, et al. (2020) Structured sampling of olfactory input by the fly mushroom body. BioRxiv.

73. Jansen J, Brodal A (1940) Experimental studies on the intrinsic fibers of the cerebellum. II. The cortico-nuclear projection. J Comp Neurol 73(2):267-321.

74. Chambers WW, Sprague JM (1955) Functional localization in the cerebellum. I. Organization in longitudinal cortico-nuclear zones and their contribution to the control of posture, both extrapyramidal and pyramidal. J Comp Neurol 103(1):105-129.

75. Chambers WW (1955) Functional localization in the cerebellum. AMA Arch NeurPsych 74(6):653.

76. Heffley W, et al. (2018) Coordinated cerebellar climbing fiber activity signals learned sensorimotor predictions. Nat Neurosci 21(10):1431-1441.

77. Carta I, Chen CH, Schott AL, Dorizan S, Khodakhah K (2019) Cerebellar modulation of the reward circuitry and social behavior. Science 363(6424).

78. Kostadinov D, Beau M, Blanco-Pozo M, Häusser M (2019) Predictive and reactive reward signals conveyed by climbing fiber inputs to cerebellar Purkinje cells. Nat Neurosci 22(6):950-962.

79. Larry N, Yarkoni M, Lixenberg A, Joshua M (2019) Cerebellar climbing fibers encode expected reward size. Elife 8.

80. Sendhilnathan N, Semework M, Goldberg ME, Ipata AE (2020) Neural Correlates of Reinforcement Learning in Mid-lateral Cerebellum. Neuron 106(1):188-198.e5.

81. Guenthner CJ, Miyamichi K, Yang HH, Heller HC, Luo L (2013) Permanent genetic access to transiently active neurons via TRAP: targeted recombination in active populations. Neuron 78(5):773-784.

82. Wickersham IR, Sullivan HA, Seung HS (2010) Production of glycoprotein-deleted rabies viruses for monosynaptic tracing and high-level gene expression in neurons. Nat Protoc 5(3):595-606.

83. Paxinos G, Franklin KBJ (2013) The Mouse Brain in Stereotaxic Coordinates 4th ed 31231.

84. Pnevmatikakis EA, Giovannucci A (2017) NoRMCorre: An online algorithm for piecewise rigid motion correction of calcium imaging data. J Neurosci Methods 291:8394. 


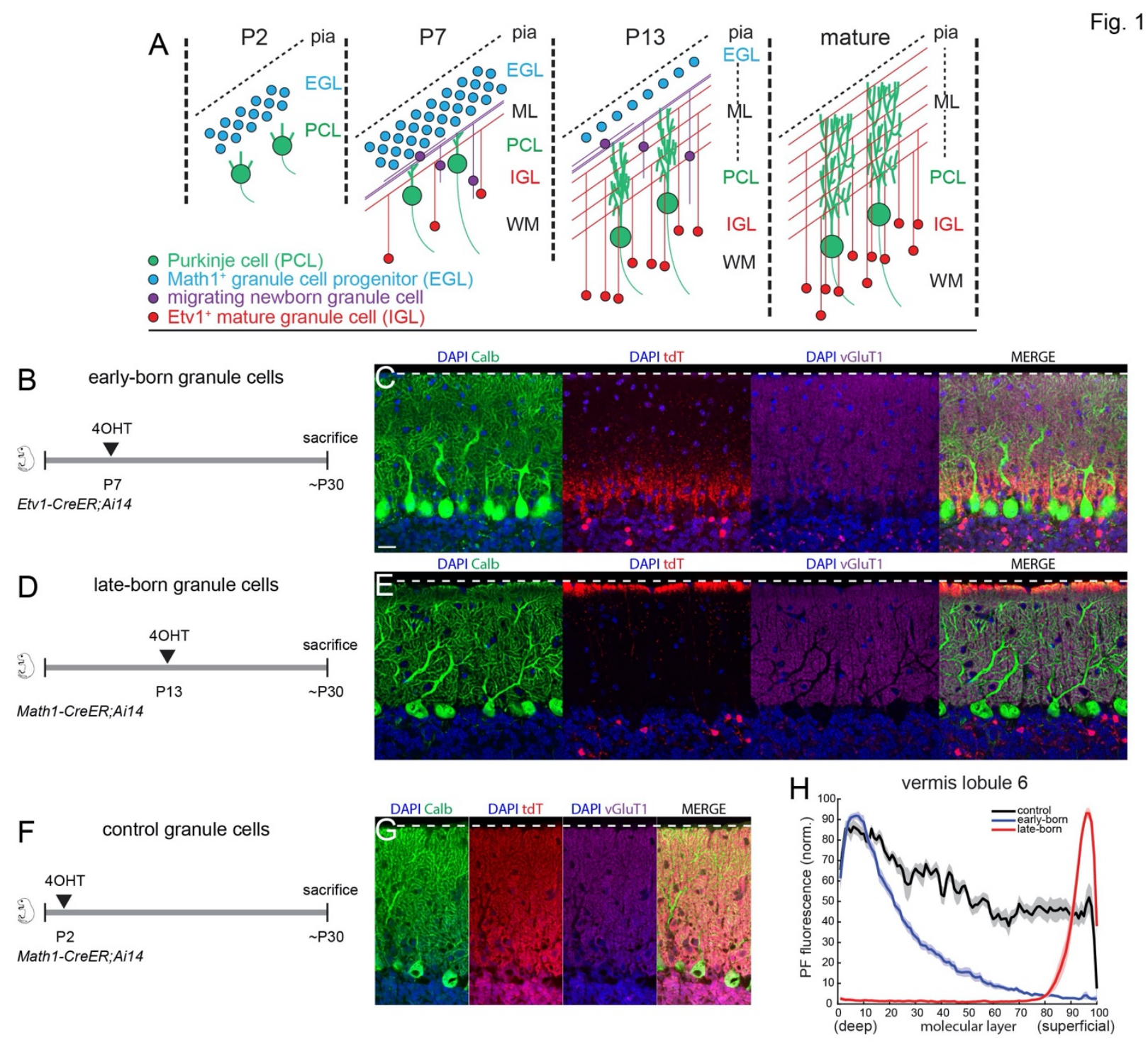

Fig. 1. Genetic strategies for accessing birth timing-defined cerebellar granule cells (GrCs). (A) Schematic summary of postnatal GrC development. Math1 is expressed exclusively in GrC progenitors, and Etv1 in postmitotic GrCs. EGL, external granule cell (germinal) layer; pia, pia mater; ML, molecular layer; PCL, Purkinje cell layer; IGL, internal granule cell layer; WM, white matter layer. $(\mathrm{B}-\mathrm{G})$ Genetic strategies and corresponding images showing parallel fiber (PF) innervation of the cerebellar molecular layer for access to early-born $(\mathrm{B}, \mathrm{C})$, late-born (D, E), and control (F, G) GrCs. Images were taken from vermis lobule 6. Blue, DAPI; green, calbindin, a marker of Purkinje cells; red, tdTomato from the Cre reporter Ai14; purple, vGluT1, a marker of PF synapses. Dotted white lines indicate pia surface. Scale bar, $20 \mu \mathrm{m}$. (H) Quantification of PFderived fluorescence across the depths of the molecular layer in vermis lobule $6 . \mathrm{N}=12$ sections from 2 mice each. 

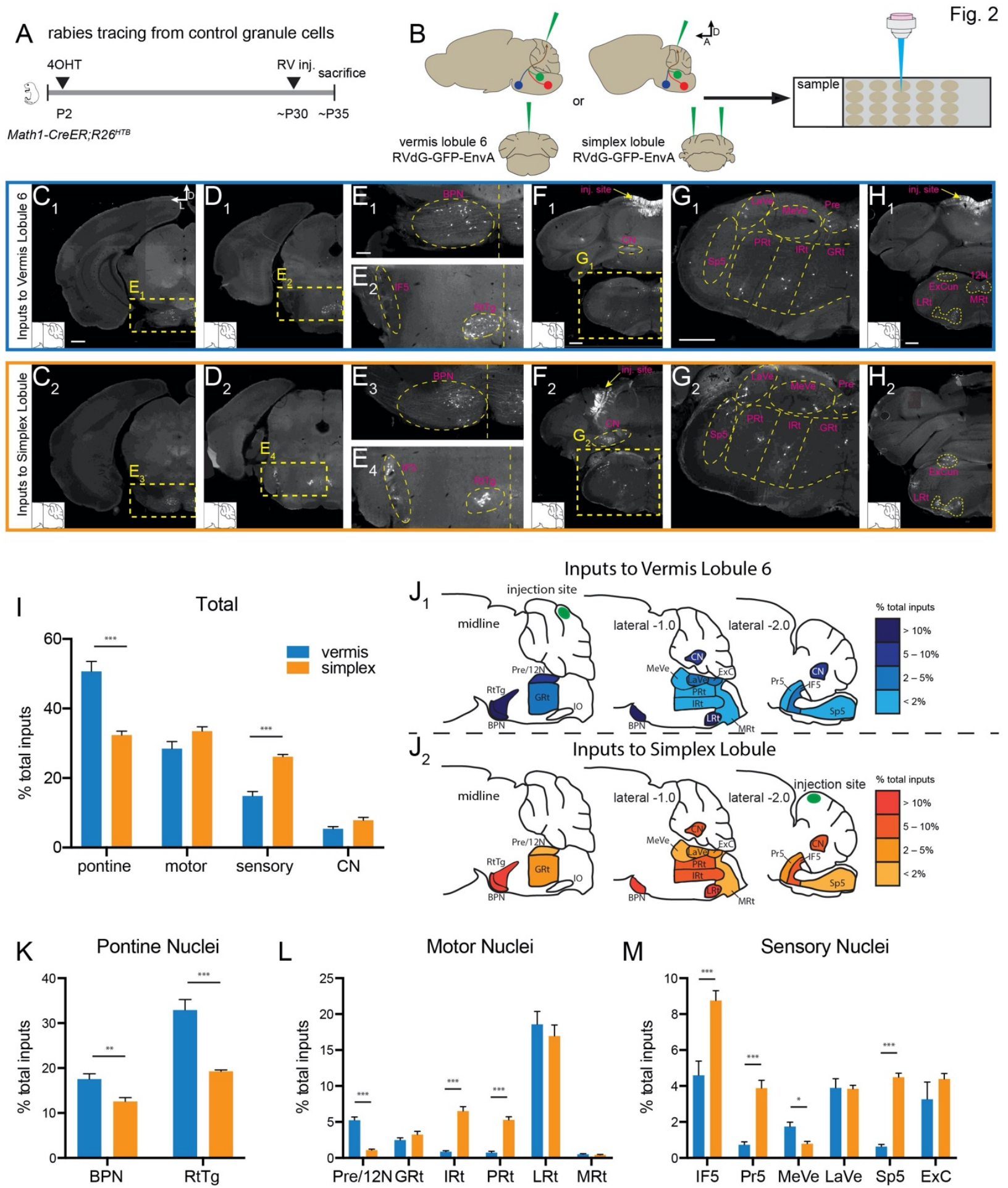

Fig. 2. Rabies tracing of mossy fiber inputs to GrCs of vermis lobule 6 and simplex. (A, B) Time course (A) and schematic (B) of retrograde monosynaptic rabies tracing. RVdG-GFP-EnvA, rabies virus deleted for glycoprotein, expressing GFP, and pseudotyped with EnvA. D, dorsal; A, anterior. Top, sagittal view; bottom, coronal view. $(\mathrm{C}-\mathrm{H})$ Example GFP-labeled input cells to vermis lobule $6\left(\mathrm{C}_{1}-\mathrm{H}_{1}\right)$ and simplex $\left(\mathrm{C}_{2}-\mathrm{H}_{2}\right)$ GrCs. D, dorsal; L, lateral. Scale bars, $500 \mu \mathrm{m}$ for C, D, F, G, H; $200 \mu \mathrm{m}$ for E. Insets at the bottom left show section planes in sagittal atlas. (I) Total 
distribution of presynaptic inputs from super-categorized brainstem nuclei to vermis lobule 6 and simplex GrCs. (J) Schematic summary of distribution of presynaptic inputs from brainstem nuclei to vermis lobule 6 and simplex GrCs. $(\mathrm{K}-\mathrm{M})$ Distribution of inputs from brainstem pontine $(\mathrm{K})$, motor (L), and sensory (M) nuclei to vermis lobule 6 and simplex GrCs. $\mathrm{N}=8$ (vermis), 8 (simplex). Error bars, SEM. ${ }^{*} p<0.05,{ }^{* *} p<0.01,{ }^{* * *} p<0.001$ (multiple unpaired t tests with Bonferroni correction). BPN, basal pontine nucleus; $\mathrm{CN}$, cerebellar nuclei; ExC, external cuneate nucleus GRt, gigantocellular reticular nucleus; IF5, interfascicular trigeminal nucleus; IRt, intermediate reticular nucleus; LaVe, lateral vestibular nuclei; LRt, lateral reticular nucleus; MeVe, medial vestibular nuclei; MRt, medial reticular nucleus; Pr5, principle trigeminal sensory nucleus; Pre/12N, prepositus/hypoglossal nucleus; PRt, parvicellular reticular nucleus; RtTg, reticulotegmental nucleus of the pons; Sp5, spinal trigeminal nucleus. 
A

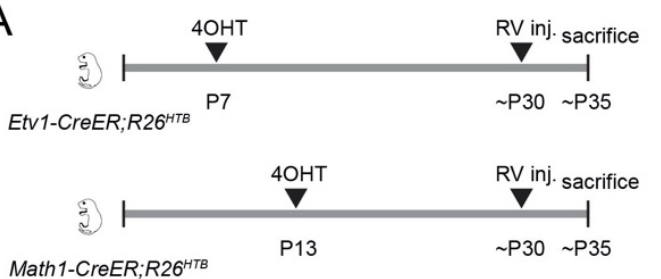

vermis lobule 6

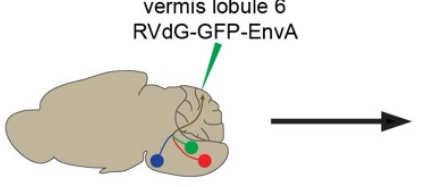

C

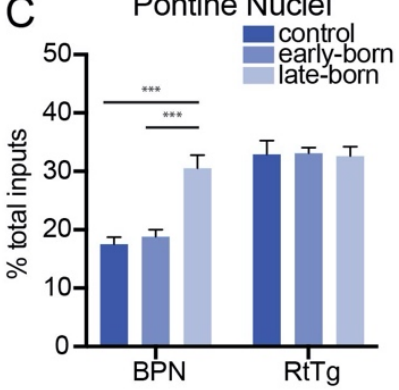

B

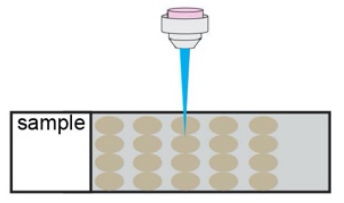

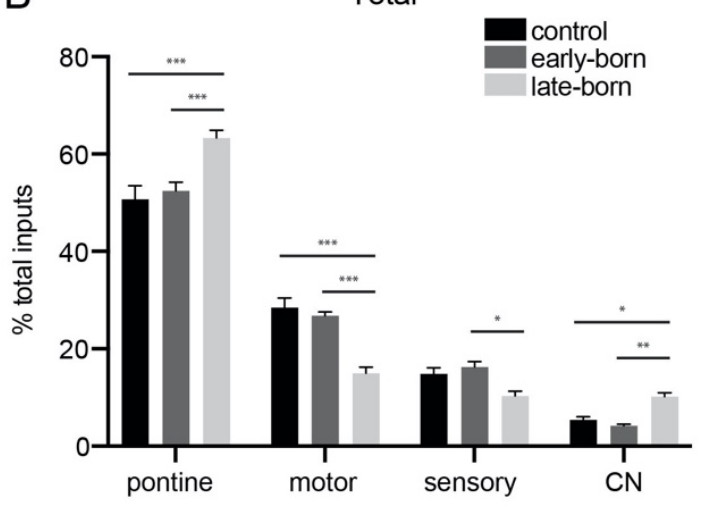

Fig. 3. Rabies tracing of mossy fiber inputs to birth timing-defined vermis lobule 6 GrCs. (A) Schematic of experimental procedures; retrograde monosynaptic rabies tracing using RVdG-GFPEnvA, rabies virus deleted for glycoprotein, expressing GFP, and pseudotyped with EnvA. (B) Total distribution of presynaptic inputs from super-categorized brainstem nuclei to control, earlyborn and late-born GrCs of vermis lobule 6. (C-E) Distribution of presynaptic inputs from brainstem pontine (C), motor (D), and sensory (E) nuclei to control, early-born and late-born GrCs of vermis lobule $6 . \mathrm{N}=8$ (control), 8 (early-born), 10 (late-born). Error bars, SEM. ${ }^{*} p<0.05,{ }^{* *} p<$ $0.01,{ }^{* * *} p<0.001$ (ordinary two-way ANOVA with Tukey's multiple comparisons test). See Figure 2 legend for anatomical abbreviations. 

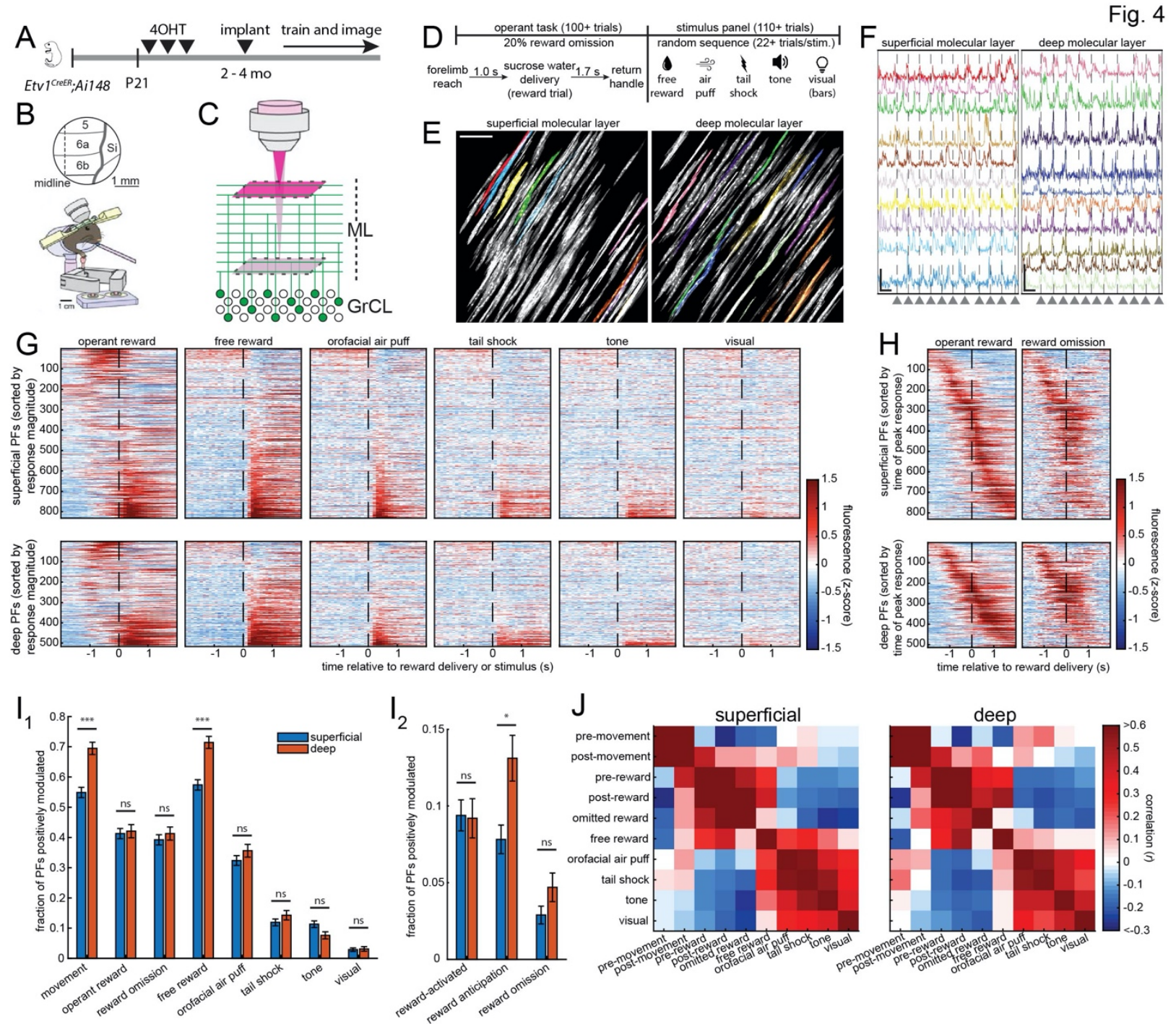

Fig. 4. Simultaneous in vivo multi-depth two-photon imaging of deep and superficial parallel fibers (PFs) during an operant task and in response to a stimulus panel. (A) Genetic strategy for depth-unbiased GCaMP6 expression in PFs. (B) Window implantation. 5, vermis lobule 5; 6a/b, vermis lobule $6 \mathrm{a} / \mathrm{b}$; Si, simplex lobule. (C) Optical strategy for multi-depth two-photon nearsimultaneous imaging of superficial and deep PFs, corresponding to late- and early-born GrCs, respectively. ML, molecular layer; GrCL, granule cell layer. (D) Operant task structure and stimulus panel with appetitive, aversive, auditory, and visual stimuli. (E) Example superficial and deep imaging fields-of-view with active PFs. Scale bar, $40 \mu \mathrm{m}$. (F) Example panels of $\mathrm{PF} \mathrm{Ca}^{2+}$ responses from fields-of-view in panel D across several trials of the operant task. Gray arrowheads and vertical dashed lines denote reward onset. Vertical scale bars, 5 S.D. (standard deviation); horizontal scale bars, 5 seconds. (G, H) Heat plots of trial-averaged $\mathrm{Ca}^{2+}$ traces of individual PFs temporally aligned to reward delivery $(\mathrm{G}, \mathrm{H})$ or stimulus $(\mathrm{G})$ onset and sorted by magnitude of response to operant reward or stimulus $(\mathrm{G})$ or time of peak response during operant task trials $(\mathrm{H})$ (831 superficial and 511 deep PFs from 19 sessions from 5 mice). (I) Fraction of PFs at both depths significantly positively modulated by each task variable or stimulus $\left(\mathrm{H}_{1} ; * * *, p<5 \times 10^{-6}\right.$ corrected; Wilcoxon rank-sum test with Holm-Bonferroni correction, $\mathrm{n}=19$ sessions), or by reward, reward anticipation, or reward omission $\left(\mathrm{H}_{2} ;{ }^{*}, p<0.05\right.$ corrected; Wilcoxon rank-sum 
test with Holm-Bonferroni correction, $\mathrm{n}=19$ sessions). See Methods. (J) Linear regression analysis to explain each individual PF's time-varying fluorescence as a weighted sum of behavioral events and stimuli (see Methods). Matrix shows the correlation coefficient between the weights assigned to each pairing of regressors across all PFs from either depth. 

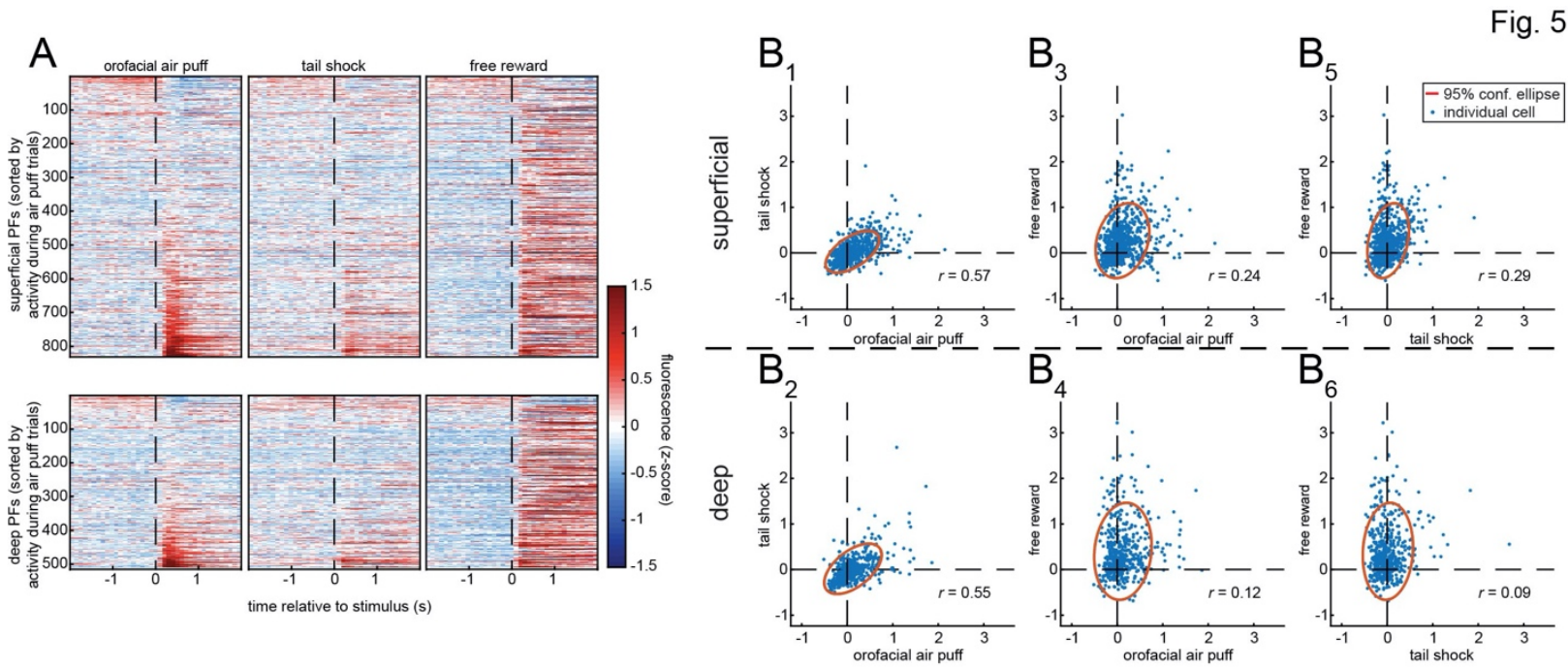

Fig. 5. Evidence for GrC encoding of aversive stimuli. (A) Heat plot of trial-averaged $\mathrm{Ca}^{2+}$ traces of individual parallel fibers (PFs) temporally aligned to air puff and tail shock and sorted by magnitude of response to air puff (831 superficial and 511 deep PFs from 19 sessions from 5 mice). (B) Following the analysis in Fig. 4J, for superficial (top) or deep PFs (bottom), correlations between the linear regression weight assigned to air puff versus tail shock $\left(\mathrm{B}_{1}, \mathrm{~B}_{2}\right)$, air puff versus free reward $\left(B_{3}, B_{4}\right)$, and tail shock versus free reward $\left(B_{5}, B_{6}\right)$. Each blue dot is a PF $(831$ superficial PFs, 511 deep PFs), and each red ellipse denotes 95\% confidence. Pearson correlation coefficients $(r)$ are displayed. 


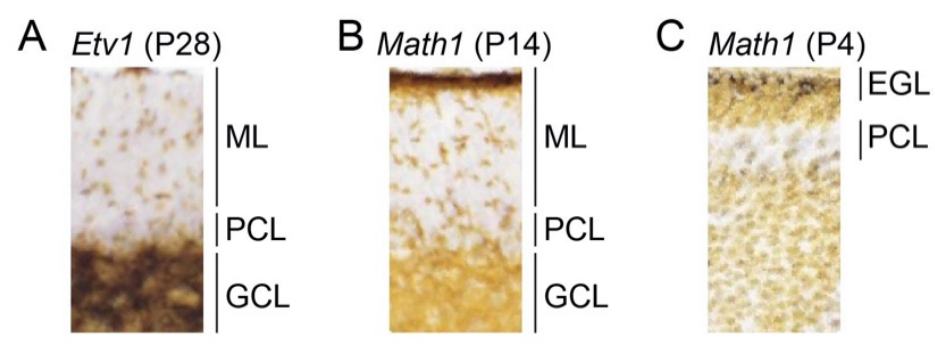

Fig. S1

Fig. S1. Expression patterns of transcription factors Etv1 and Math1. (A-C) In situ hybridization of Etv1 at postnatal day 28 (P28, A), Math1 at P14 (B), and Math1 at P4 (C) in cerebellar cortex vermis lobule 6. Data are from Allen Brain Atlas (40). ML, molecular layer; PCL, Purkinje cell layer; GCL, granule cell layer; EGL, external germinal layer. 

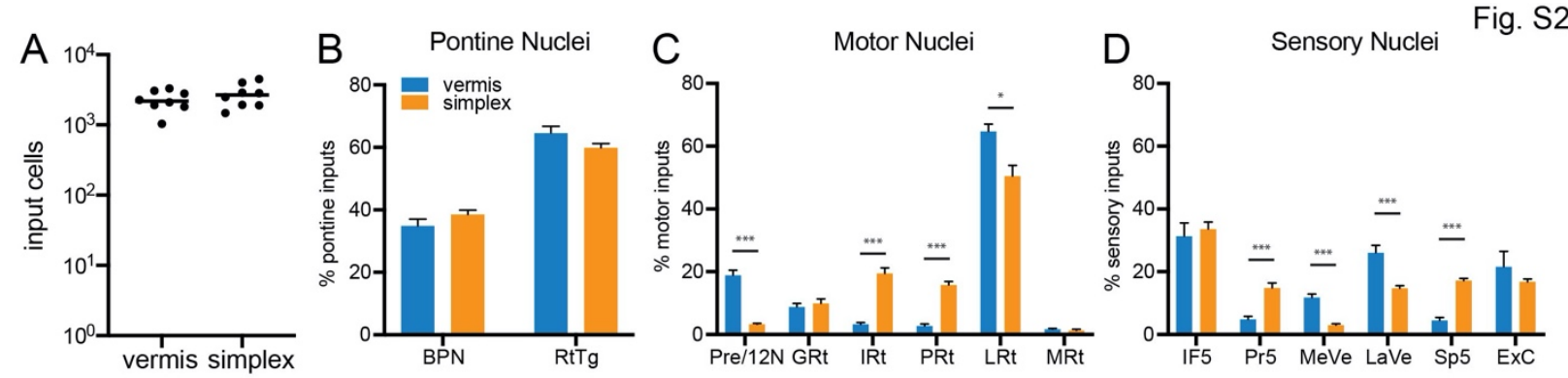

Fig. S2. Additional analysis of mossy fiber inputs to granule cells (GrCs) of vermis lobule 6 and simplex. (A) Number of input cells to vermis lobule 6 and simplex control GrCs not defined by birth timing. (B-D) Proportion of inputs to vermis lobule 6 and simplex control GrCs contributed by each nucleus to total inputs from brainstem pontine (B), motor (C), and sensory (D) regions. $\mathrm{N}=8$ (vermis), 8 (simplex). Error bars, SEM. ${ }^{*} p<0.05,{ }^{* *} p<0.01,{ }^{* * *} p<0.001$ (multiple unpaired $t$ tests with Bonferroni correction). See Figure 2 legend for anatomical abbreviations. 

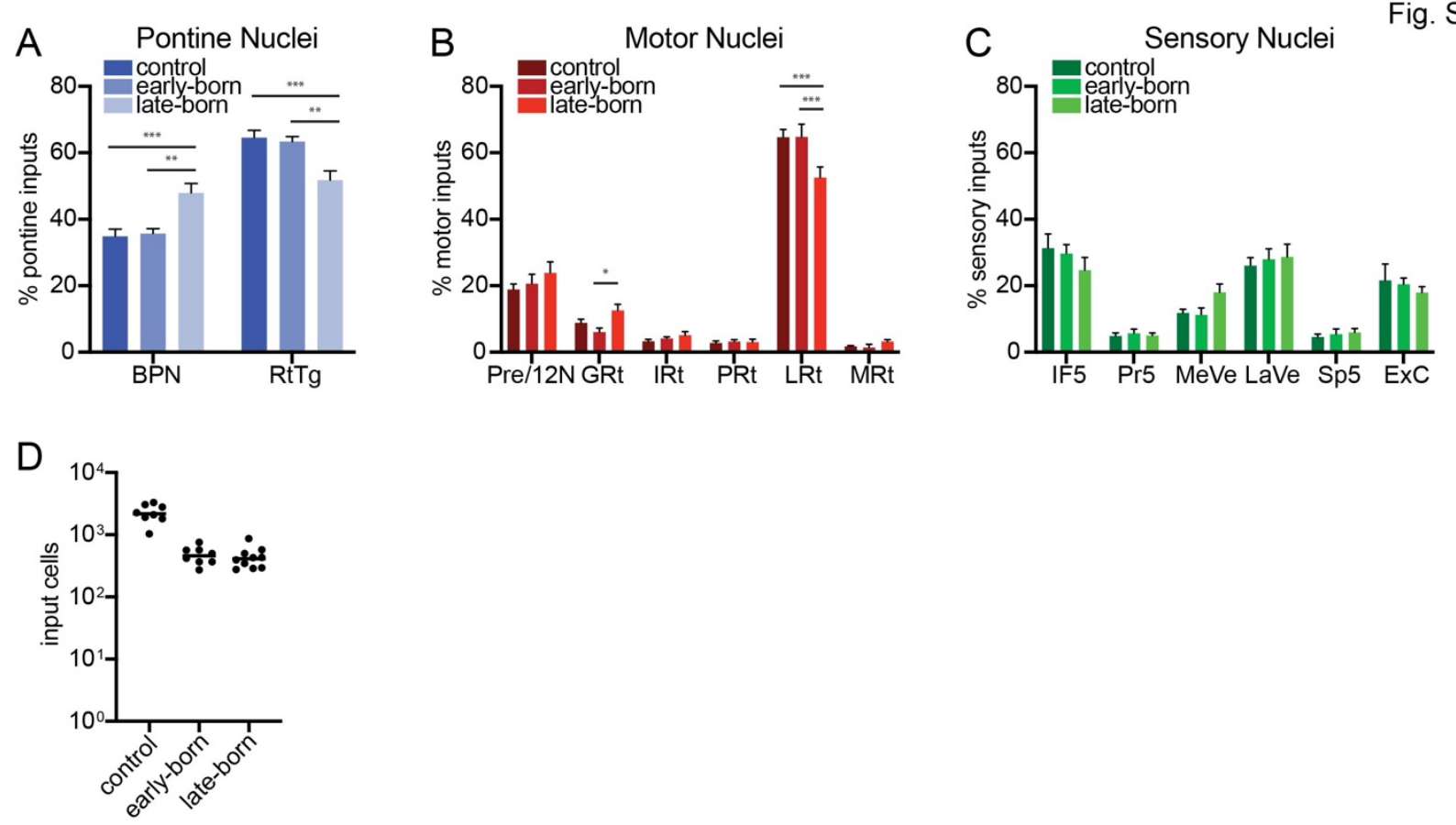

Fig. S3. Additional analysis of mossy fiber inputs to birth timing-defined vermis lobule 6 GrCs. (A-C) Proportion of inputs to vermis lobule 6 control, early-born, and late-born GrCs contributed by each nucleus to total inputs from brainstem pontine (B), motor (C), and sensory (D) regions. $\mathrm{N}=8$ (control), 8 (early-born), 10 (late-born). Error bars, SEM. ${ }^{*} p<0.05,{ }^{* *} p<$ $0.01,{ }^{* * *} p<0.001$ (ordinary two-way ANOVA with Tukey's multiple comparisons test). (D) Number of input cells to vermis lobule 6 control, early-born, and late-born GrCs. See Figure 2 legend for anatomical abbreviations. 

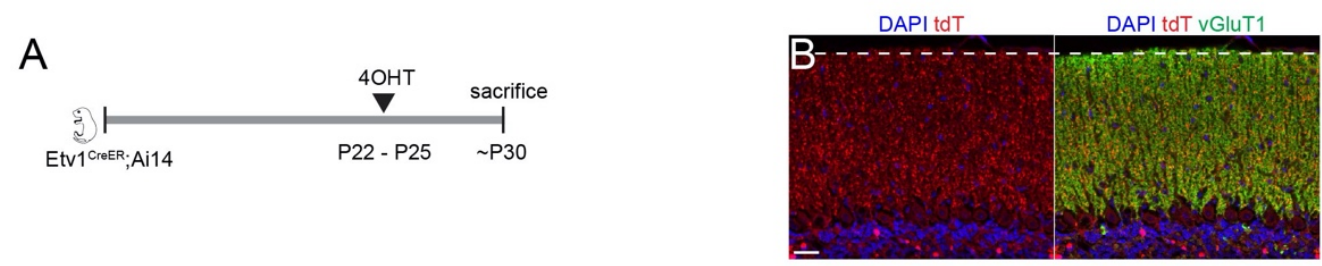

Fig. S4
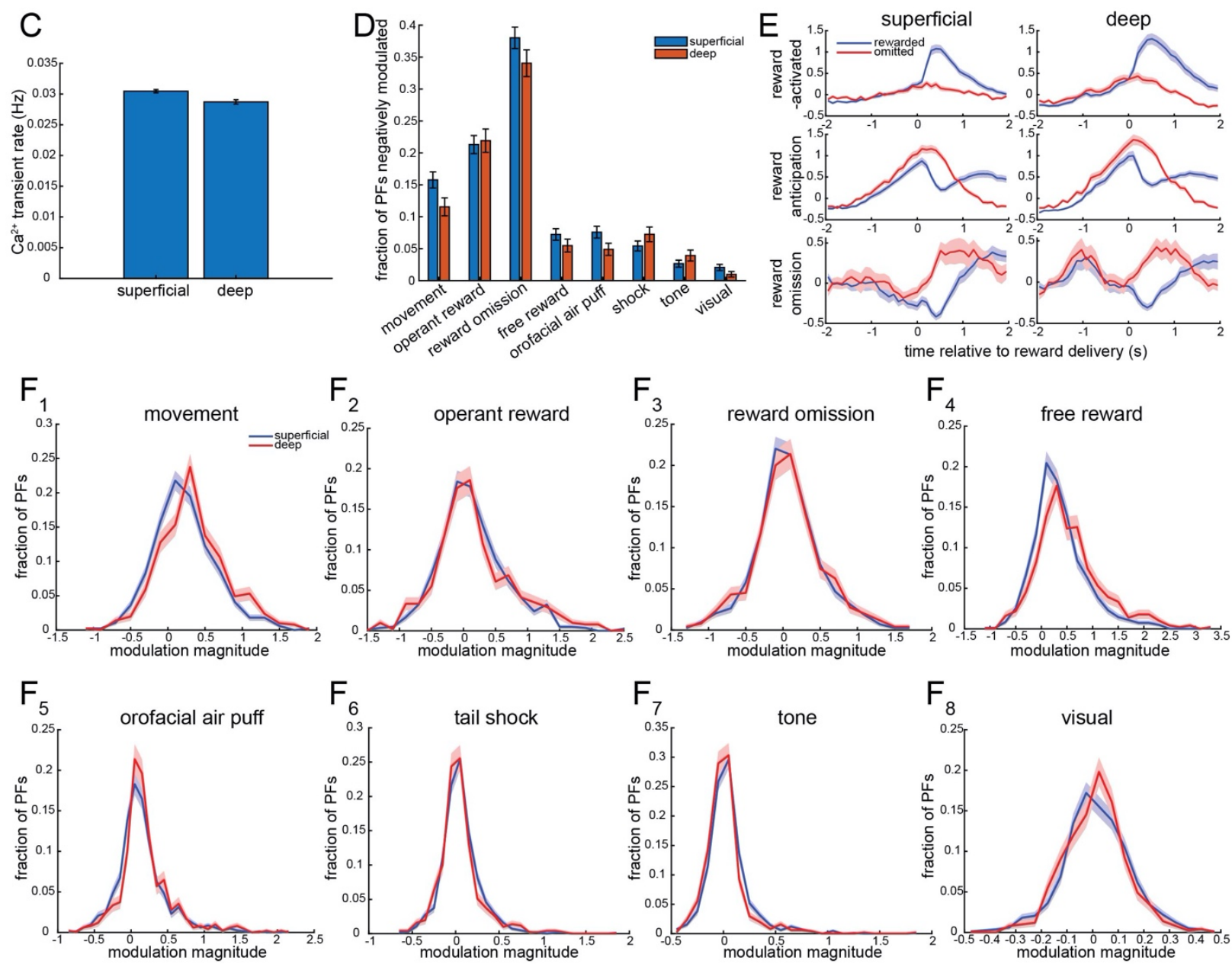

Fig. S4. Additional analysis of multi-depth two-photon imaging data, Part I. (A) Genetic strategy for expressing GCaMP6f sparsely in GrCs not defined by birth timing, producing PFs that span all depths. (B) Fluorescence images showing PF innervation of the cerebellar molecular layer in a sparse and birth-timing agnostic manner. Image was taken from vermis lobule 6a. Scale bar, $20 \mu \mathrm{m}$. (C) $\mathrm{Ca}^{2+}$ transient rates of PFs at both depths. (D) Fraction of PFs at both depths significantly negatively modulated by each task variable or stimulus. Error bars, SEM; all pairs ns, $p>0.05$ (Wilcoxon rank-sum test with Holm-Bonferroni correction, $\mathrm{n}=19$ sessions). (E) Averaged traces of PFs at both depths identified as reward-activated, reward anticipation, or reward omission PFs. (F) Distribution of modulation magnitudes of PFs at both depths by each task variable or stimulus. 

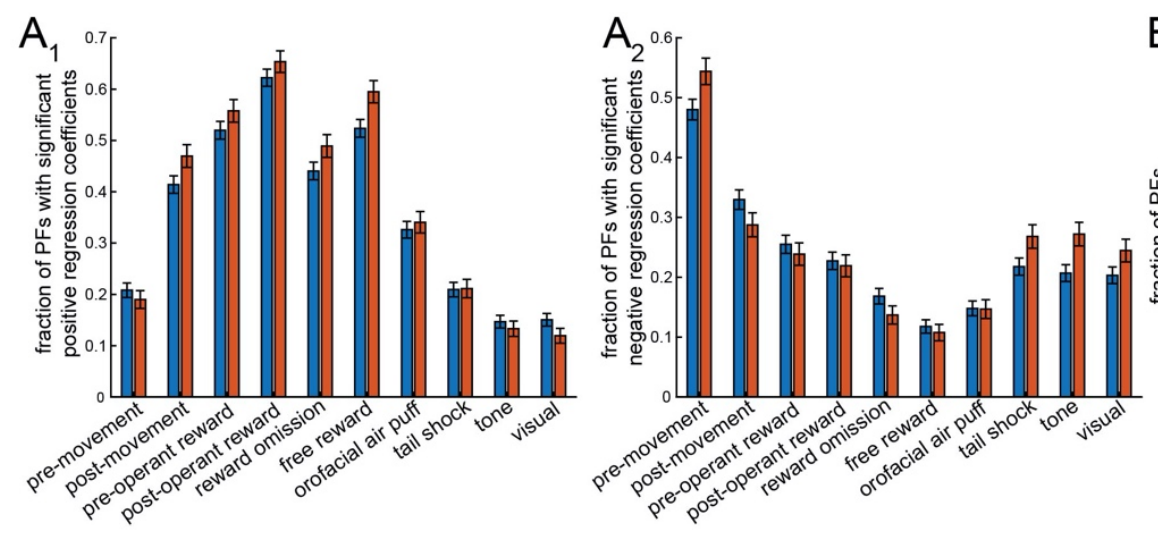

B

Fig. S5
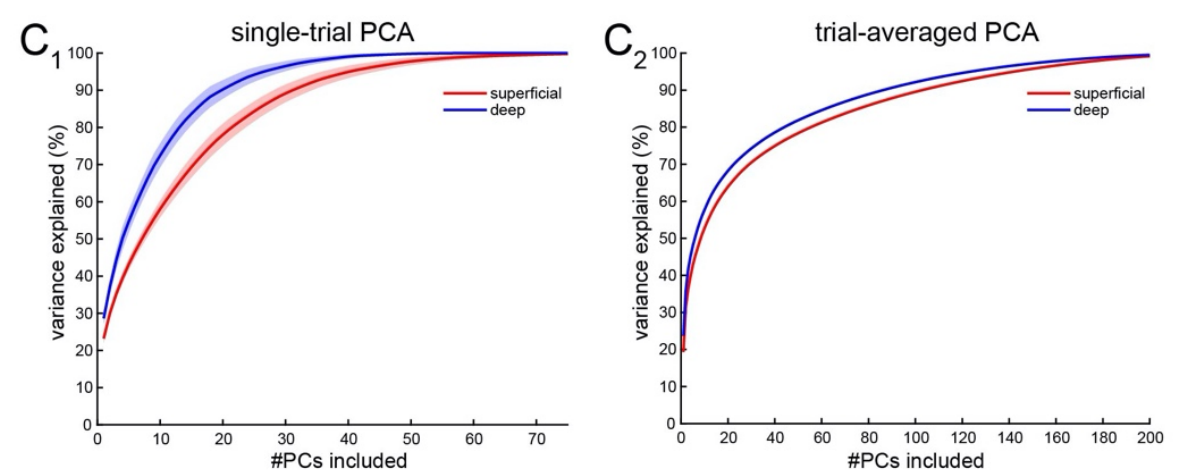

Fig. S5. Additional analysis of multi-depth two-photon imaging data, Part II. (A) Using the regression analysis described in Fig. 4J, fraction of PFs at both depths with significant positive $\left(A_{1}\right)$ or negative $\left(A_{2}\right)$ regressors for each task variable or stimulus. Error bars, SEM; all pairs ns, $p>0.05$ (Wilcoxon rank-sum test with Holm-Bonferroni correction, $\mathrm{n}=19$ sessions). (B) Distribution of number of significant regressors of PFs at both depths. Error bars, SEM (n = 19 sessions). (C) Principal components analysis (PCA) of superficial and deep PF ensemble activity based on dimensionality computed from single-trial $\left(\mathrm{C}_{1}\right.$; mean $\pm \mathrm{SEM}, \mathrm{n}=19$ sessions $)$ or trialaveraged $\left(\mathrm{C}_{2}\right)$ activity of all superficial or deep PFs in all imaging sessions (see Methods). In both cases, about 5-8 dimensions are needed to explain half of the ensemble variance. 
Movie 1: Multi-depth, near-simultaneous $\mathrm{Ca}^{2+}$ imaging of parallel fibers in the superficial (left) and deep (right) molecular layer during operant task. Movie is $4 \times$ temporally downsampled and is thus played at $4 \mathrm{x}$ speed. Original frame rate is $9.75 \mathrm{~Hz}$. 